\title{
Análise de Sentimentos em Tweets em Português Brasileiro
}

\author{
Daniel P. Kansaon ${ }^{1}$, Michele A. Brandão ${ }^{2}$, Saulo A. de Paula Pinto ${ }^{1}$ \\ ${ }^{1}$ Pontifícia Universidade Católica de Minas Gerais (PUC-MG) - Belo Horizonte, MG - \\ Brasil \\ ${ }^{2}$ Universidade Federal de Minas Gerais (UFMG) - Belo Horizonte, MG - Brasil \\ 1\{daniel.kansaon,saulo\}@sga.pucminas.br, ${ }^{2}$ micheleabrandao@dcc.ufmg.br

\begin{abstract}
There are several studies on sentiment analysis for the English language. In the case of Brazilian Portuguese, the number of papers is smaller because there are not so many datasets available and methods to perform the analysis. This work presents a methodology to compare techniques that classify feelings expressed directly or indirectly in tweets in the Brazilian Portuguese language. In addition, seven classes of feelings are considered and identified in the tweets. The results are promising when classifying distinct feelings, as the best classifier achieves $85 \%$ of accuracy. On the other hand, relations between close feelings present results less than $70 \%$ of accuracy.
\end{abstract}

Resumo. Existem diversos trabalhos sobre análise de sentimentos para a língua inglesa. No caso do português brasileiro, a quantidade de trabalhos é menor por não existirem tantas bases de dados disponíveis e métodos para realizar a análise. Este trabalho apresenta uma metodologia para comparar técnicas que classificam sentimentos expressos diretamente ou indiretamente em tweets no idioma português brasileiro. Ademais, são consideradas e identificadas sete classes de sentimentos nos tweets. Os resultados são promissores ao classificar sentimentos distintos, pois o melhor classificador alcança $85 \%$ de acerto. Por outro lado, relações entre sentimentos próximos apresentam resultados inferiores a $70 \%$ de acerto.

\section{Introdução}

Dados são coleções de valores que podem ser explorados e processados a fim de se obter padrões, associações, mudanças e anomalias em prol de algum objetivo. Nas últimas décadas, devido à globalização, a produção de dados na forma digital tem aumentado exponencialmente. Algumas estimativas apontam que a quantidade de informação produzida no mundo se duplica a cada vinte meses [Dwivedi et al., 2016]. Um bom processamento e manipulação dessas informações pode trazer benefícios a organizações, grupos de investidores e até mesmo pessoas que desejam extrair algum tipo de informação sobre um conjunto de dados [Dwivedi et al., 2016].

Com o avanço tecnológico e a popularização das redes sociais online, as pessoas utilizam cada vez mais essas redes como um grande aliado para a comunicação, fazendo com que as redes sejam uma grande fonte de informação. Devido a grande quantidade de dados disponíveis, as análises dessas redes podem trazer informações valiosas para as várias empresas que buscam informações sobre seus produtos, clientes e que procuram entender melhor o mercado e os concorrentes, por exemplo. 
Uma das técnicas que auxiliam essas avaliações é a análise de sentimentos, que tem como ideia principal extrair e descobrir qual o sentimento expresso em um texto. Assim, é possível classificar a sua polaridade, que é a tarefa de determinar se um sentimento expresso em um texto é positiva, negativa ou até mesmo neutra [Cavalcanti et al., 2012]. Ademais, a análise de sentimentos é uma das técnicas aplicadas no contexto das redes sociais e está associada com a mineração de dados. Essa técnica pode ser empregada em diversas situações, principalmente, para entender o senso comum sobre um determinado acontecimento, como: eventos, economia e política.

Nesse contexto, este trabalho apresenta uma análise de sentimentos expressos pelos usuários em tweets, postagens realizadas no Twitter. A identificação dos sentimentos tem como apoio processos de mineração, que visam extrair informações relevantes, utilizando-se de métodos de classificação para determinar o sentimento expresso nos tweets. Assim, o foco deste trabalho é responder à pergunta: quais algoritmos de classificação podem melhor descobrir sentimentos em tweets em português brasileiro? Em outras palavras, uma pesquisa exploratória é realizada com objetivo principal de verificar o desempenho de diferentes algoritmos de classificação ao serem aplicados na análise de sentimentos semelhantes e distintos em tweets em português brasileiro.

O restante deste artigo está organizado da seguinte forma. A Seção 2 apresenta os trabalhos relacionados. A Seção 3 descreve a metodologia utilizada e a Seção 4 descreve os resultados obtidos nos experimentos, detalhando a aplicação de cada método. Já a Seção 5 discute os resultados experimentais obtidos. Finalmente, a Seção 6 apresenta conclusões e comenta sobre trabalhos futuros.

\section{Trabalhos Relacionados}

A mineração de dados juntamente com a análise de sentimentos tem ampla aplicabilidade em várias áreas do conhecimento, por exemplo, jogos, educação e política, e sempre busca informações significativas em grandes volumes de dados. Assim, existem diversos trabalhos recentes que enfatizam o uso da mineração de dados, especificamente, a aplicação de técnicas de classificação.

Nesse cenário, Bouazizi e Ohtsuki [2016] propõem uma abordagem para quantificação de sentimentos no Twitter. Tal trabalho destaca a importância da detecção de sentimentos em tweets e apresenta uma maneira de extrair diferentes sentimentos utilizando a técnica de classificação Random Forest. Foram coletados cerca de 40.000 tweets e atribuídos um sentimento para cada um, sendo eles: amor, felicidade, diversão, entusiasmo, alívio, ódio, raiva, tristeza, tédio preocupação, surpresa e neutralidade.

Ademais, Pandeye e Rajpoot [2016] analisam sentimentos através da comparação de diferentes técnicas de classificação como J48, Random Forest, Decision Tree, Random Tree, NaiveBayes, SimpleNaiveBayes, NaiveBayes e DecisionStump. Essa análise foi feita com um conjunto de dados sobre o consumo de álcool por estudantes de uma escola. O algoritmo que realizou a melhor classificação foi Decision Stump, com um resultado de 95,44\% de acurácia. Além disso, Caetano et al. [2017] utilizaram a ferramenta SentiStrength para analisar sentimentos no Twitter sobre candidatos das eleições americanas. Tal estudo foi realizado com o objetivo de analisar 
Tabela 1. Modelos diferentes de emoções básicas propostas por teóricos. Fonte: adaptada de Yadollahi et al. [2017].

\begin{tabular}{|l|c|l|}
\hline Teórico & Ano & \multicolumn{1}{c|}{ Emoções Básicas } \\
\hline Ekman & 1972 & raiva, desgosto, medo, alegria, tristeza, surpresa \\
\hline Plutchik & 1986 & $\begin{array}{l}\text { raiva, antecipação, desgosto, medo, alegria, tristeza, surpresa, } \\
\text { confiança }\end{array}$ \\
\hline Shaver & 1987 & raiva, medo, alegria, amor, tristeza, surpresa \\
\hline Lovheim & 2011 & $\begin{array}{l}\text { raiva, desgosto, angústia, medo, alegria, interesse, vergonha, } \\
\text { surpresa }\end{array}$ \\
\hline
\end{tabular}

a homofilia (a tendência de pessoas se relacionarem com semelhantes) entre usuários. Já Garimella et al. [2018] realizaram um estudo a fim de quantificar controvérsias em redes sociais baseada em gráficos, tal estudo indica que os gráficos que apresentam temas controversos, possuem uma estrutura de cluster, onde indivíduos com opinião similar tendem a ampliar os argumentos um dos outros.

Em relação à comparação de diferentes métodos de análise sentimentos, Araújo et al. [2015] apresentam um estudo que compara oito diferentes métodos para detecção de sentimentos em tweets no idioma inglês. Já Araújo et al. [2016] propôs uma análise de sentimento multilíngue, onde é feita tradução automática de textos para o inglês, e posteriormente utiliza-se métodos existentes na língua inglesa para a análise de sentimento. Ademais, Yadollahi et al. [2017] realizam uma categorização cuidadosa de tarefas na análise de sentimentos e retratam a importância de uma taxonomia clara e lógica dentro dessa análise. Adicionalmente, métodos e melhorias do estado da arte para a análise de sentimentos em textos foram discutidos, além de aspectos teóricos ligados ao surgimento das emoções e sentimentos. Note que os sentimentos estão relacionados às emoções visto que são respostas a elas.

Nesse contexto, a Tabela 1 apresenta algumas emoções básicas que podem ser encontradas em todos os seres humanos, bem como a respectiva classificação de acordo com as teorias da emoção [Yadollahi et al., 2017]. Além disso, Bouazizi e Ohtsuki [2016] também classificam sentimentos considerando um conjunto de classes como: amor, feliz, tristeza e raiva. A análise de tais estudos auxiliou na escolha de sete sentimentos para identificar em tweets coletados neste trabalho: Triste, Chateado, Amor, Feliz, Raiva, Inveja, Ironia.

Em relação à comparação de várias técnicas de classificação, Garg e Khurana [2014] propõem um estudo em um contexto diferente da análise de sentimentos. O objetivo é utilizar algoritmos de classificação para projetar um modelo eficaz de detecção de intrusão, impedindo que as redes de computadores sejam invadidas. Os métodos foram avaliados usando 41 atributos e cerca de 94.000 instâncias para o conjunto de treinamento, além de 48.000 instâncias para o conjunto de teste. Por fim, foi apresentada uma lista de 45 métodos de classificação ordenados pelos seus respectivos resultados. Os cinco algoritmos com melhores resultados são Rotation Forest, Random Tree, Random Committee, Random Forest e IBK. 


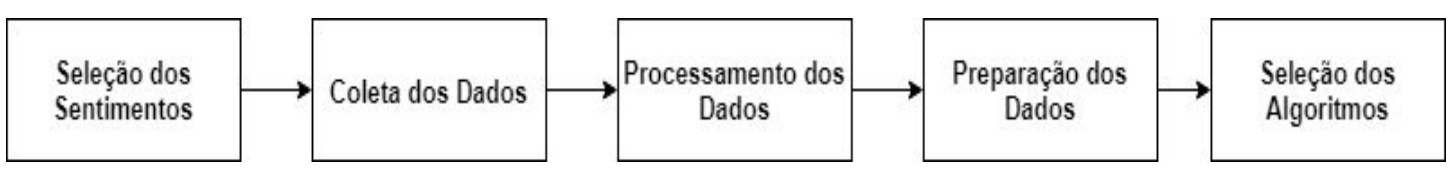

Figura 1. Etapas da metodologia.

Todos esses estudos mostram desempenhos diferentes para algoritmos de classificação a depender da base de dados utilizada. Assim, este trabalho contribui com o estado da arte ao considerar o idioma português brasileiro para realizar análise de sentimentos em tweets. Considerar tal idioma não é muito comum por não existirem muitas bases de dados disponíveis [Neri et al., 2012], além de ter poucos métodos e ontologias disponíveis no português brasileiro para realizar análises do texto. Outra contribuição é a metodologia proposta, que inclui coleta e processamento dos dados, identificação de diferentes classes de sentimentos, seleção dos algoritmos de classificação e descrição do treinamento dos classificadores.

\section{Metodologia}

Esta seção apresenta os processos de coleta, processamento e preparação dos dados para a realização da análise de sentimentos, bem como a descrição dos algoritmos de classificação selecionados e o processo de treinamento dos classificadores. Especificamente, este trabalho identifica sentimentos e emoções expressos em tweets através de métodos de classificação. Para atingir o objetivo, foi necessário realizar uma série de etapas para adaptar os dados ao formato compatível com o ambiente WEKA, que possibilita o uso de diferentes algoritmos.

A Figura 1 exemplifica as etapas principais da metodologia. Cada etapa pode ser executada mais de uma vez. As seções a seguir detalham cada uma dessas etapas.

\subsection{Seleção dos Sentimentos}

A definição das classes de sentimentos analisados durante a pesquisa tem como base os diversos sentimentos que podem ser expressos através dos tweets. As expressões podem ser identificadas de várias formas, através de emojis, emoticons, hashtag. A hashtag é composta por uma ou algumas palavras-chave relacionadas ao assunto abordado na postagem. Elas são representadas pelo símbolo de cerquilha (\#), seguida da palavra-chave. Posteriormente, as hashtags são indexadas para utilização dos mecanismos de busca. Os emojis e emoticons são amplamente usados no Twitter e estão diretamente associados a emoções. Os emojis são exibidos como imagens claras e podem conter animações. Por outro lado, os emoticons, são formas mais simples de expressar emoção e, geralmente, são representados por uma sequência de caracteres, por exemplo: $\{:(,: D,: /\}$ [Matsuda, 2017].

Os sentimentos analisados neste trabalho foram escolhidos através do estudo de outros trabalhos apresentados na Seção 2. Além disso, o estudo feito por Yadollahi et al. [2017], oferece uma contribuição importante para a escolha dos sentimentos aqui analisados, pois apresenta as emoções básicas que podem ser encontradas nos seres humanos (Tabela 1). Dessa forma, busca-se tweets que expressem os sentimentos: Triste, Chateado, Amor, Feliz, Raiva, Inveja, Ironia. Para coletar tweets que expressem 


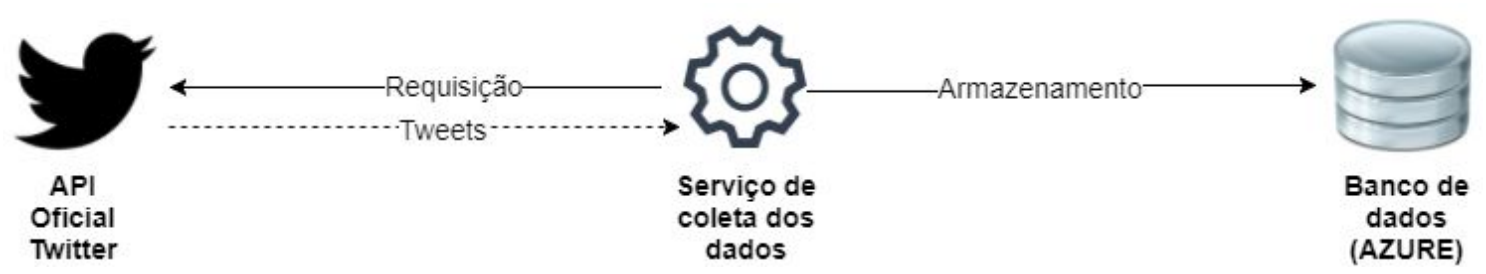

Figura 2. Detalhamento do fluxo de coleta dos dados.

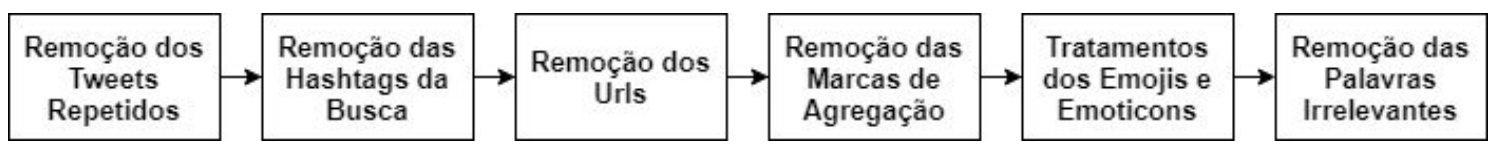

Figura 3. Etapas do processamento de cada tweet.

esses sentimentos, foi definido que o tweet precisa conter a hashtag com o nome do sentimento selecionado. Assim, são considerados os tweets que possuem as hashtags: \#Triste, \#Chateado, \#Feliz, \#Amor, \#Raiva, \#Inveja, \#Ironia.

\subsection{Coleta dos Dados}

Após a seleção dos sentimentos, foi necessário realizar a extração e o armazenamento dos dados. A API oficial do Twitter fornece vários recursos para a coleta de tweets. Por meio dela, usuários que se cadastram como desenvolvedores conseguem coletar tweets a partir de parâmetros. A API possui algumas restrições para a coleta dos dados, por exemplo, a limitação do número de cem tweets como resposta para cada consulta realizada. Apesar disso, ela se apresenta suficiente para realização do estudo. Para considerar o tweet na análise, é necessário estar no idioma português brasileiro e possuir as hashtags definidas (\#Triste, \#Chateado, \#Feliz, \#Amor, \#Raiva, \#Inveja, \#Ironia).

A Figura 2 apresenta o funcionamento do fluxo de coleta dos tweets. Toda a comunicação com a API é feita através do protocolo HTTP usando métodos de GET. Todas as respostas retornadas foram armazenadas na base de dados. A coleta foi feita entre os meses de agosto e outubro de 2017, contando com 12.814 tweets armazenados na base de dados ${ }^{1}$, criada na linguagem SQL (SQL Server) e hospedada no servidor na nuvem Azure. Por se tratar de uma plataforma gerenciada, a base de dados na nuvem oferece alta disponibilidade sem precisar da instalação de qualquer software para a utilização. Todas as informações relevantes dos tweets foram armazenadas na base de dados, sendo elas: texto, hashtag de busca, autor, data de criação, linguagem, quantidade de URLs, quantidade de menções feitas, texto do URLs e tipo.

\subsection{Processamento dos Dados}

O processamento realizado tem como objetivo tratar os dados coletados removendo as informações que não contribuem para classificação dos sentimentos. Cada tweet contém uma grande quantidade de informações que são irrelevantes para a análise. Por isso, são removidas dos tweets. Nesta etapa da metodologia, foram necessários vários passos de

\footnotetext{
${ }^{1}$ Base de dados construída a partir da coleta de tweets: http://bit.ly/dataset-analise-sentimento-BraSNAM
} 
processamento e tratamento dos dados, realizados de forma sequencial, conforme mostra a Figura 3.

\begin{tabular}{c|c|c|c|c} 
Amor & Feliz & Feliz & Amor & Triste \\
$\mathbf{x}$ & $\mathbf{x}$ & $\mathbf{x}$ & $\mathbf{x}$ & $\mathbf{x}$ \\
Triste & Triste & Chateado & Feliz & Chateado
\end{tabular}

Figura 4. Relações de sentimentos.

Na coleta dos tweets, foi observado que a API do Twitter retorna informações repetidas. Com isso, é necessário remover todos os tweets repetidos na base de dados, para que não influencie o resultado final ao aplicar os algoritmos. Em seguida, as hashtags usadas para a coleta dos tweets são removidas dos textos, os quais possuem links compartilhados e até mesmo notações utilizadas para mencionar outros usuários. Assim, as marcas de agregação identificadas através da sigla RT ou @ nome do usuário são removidas juntamente com os links encontrados. O ambiente WEKA não possui suporte a caracteres Unicode. Assim, os emojis, por se tratarem de caracteres com formato específico, são transformados em palavras-chave, formadas pela junção da letra "E" maiúscula, completada posteriormente com uma palavra que representa o respectivo símbolo do emoji, exemplo: ECoracao, EBravo, ESorridente. Por fim, as palavras conhecidas como stop words (a, as, de, para, etc) também são removidas.

Além das etapas de processamento, um critério para nivelamento dos dados é considerado com base na quantidade de tweets coletados. É necessário que os sentimentos tenham uma quantidade suficiente de exemplos ou instâncias para serem analisados, pois um número pequeno de dados pode levar a um resultado não fidedigno. Para evitar essa situação, foi definida uma premissa: um sentimento apenas é analisado, se possuir um mínimo de mil tweets. Com isso, os sentimentos: Ironia, Inveja e Raiva foram excluídos. Após a exclusão de tais sentimentos, a quantidade total de tweets disponíveis passou para 9.631, assim, representando uma média de dois mil tweets para cada sentimento restante.

\subsection{Preparação dos Dados}

Após os tratamentos realizados, os dados coletados são separados em classes de sentimentos. Algumas relações entre sentimentos são criadas para avaliar se os métodos de classificação conseguem distinguir as diferenças entre os sentimentos. As relações são formadas visando comparar os sentimentos separando em: positivos x negativos, positivos x positivos e negativos x negativos. Assim, os sentimentos amor e feliz são considerados sentimentos positivos, já os sentimentos triste e chateado, representam os sentimentos negativos. Dessa forma, a Figura 4 apresenta as relações formadas para a análise de sentimentos.

Em seguida, é necessário exportar os dados para o formato "ARFF" (Attribute Relation Format File), do ambiente WEKA. Para isso, é criado um arquivo para cada relação de sentimentos selecionados anteriormente, com os requisitos exigidos pelo WEKA. Em tal arquivo, os dados foram divididos em dois atributos, o primeiro chamado "Descricao" que contém o texto do tweet a ser analisado e o segundo atributo, chamado de "Sentimento" que define qual classe de sentimento o tweet se enquadra. 


\subsection{Seleção dos Algoritmos de Classificação e Treinamento}

Neste trabalho, os algoritmos de classificação baseados no modelo Naive Bayes são algumas das abordagens escolhidas. Esses algoritmos utilizam a probabilidade condicional para criar o modelo de dados a ser trabalhado. São considerados algoritmos como o Naive Bayes Multinominal, no qual a classe de um documento (tweets) é determinada não apenas pelas palavras existentes, mas também pela frequência que ocorrem [Witten et al., 2016]. Ademais, França e Oliveira [2014] utilizam o algoritmo Naive Bayes no idioma português brasileiro e apresentam resultados de até $90 \%$ de acurácia ao classificar polaridades expressas nos tweets relacionados aos protestos ocorridos no Brasil em 2013.

Além dos algoritmos baseados no Naive Bayes, alguns algoritmos que apresentaram bons resultados em outros trabalhos, também foram selecionados. Isso permite verificar se os resultados obtidos nos trabalhos se repetem na classificação de tweets no idioma português brasileiro. Em Garg e Khurana [2014], são avaliados o desempenho de vários métodos de classificação, conforme descrito na Seção 2. Dessa forma, foram escolhidos os algoritmos que apresentaram bons resultados e que são compatíveis com o formato dos dados trabalhados. Sendo eles: IBK, Forest e Random Committee [Garg e Khurana, 2014]. Em resumo, os algoritmos selecionados para a classificação dos tweets são: Naive Bayes, Naive Bayes Multinominal, Naive Bayes Multinominal Updateable, Sparge Generative Model, DMNB Text, Complement Naive Bayes, Bayesian Logistc Regression, IBK, Forest e Random Committee.

Para treinar o classificador, é comum usar um terço dos dados para testes e dois terços para treino, mas o problema dessa abordagem é que a parte usada para treino pode não ser representativa do problema e nem do conjunto de testes. A base de dados deve ser representada na proporção certa, pois se o problema não estiver bem representado, dificilmente qualquer algoritmo apresentará um bom resultado. Uma maneira de mitigar qualquer parcialidade causada pela amostragem, é utilizar o tipo de treinamento conhecido como validação cruzada (de dez partições ou vias). Nesse caso, os dados são divididos em dez partições de tamanhos aproximadamente iguais e cada uma delas é usada para testes e o restante é usado para treinamento. Esse processo é repetido dez vezes para que no final, cada instância seja usada uma vez para testar. Para obter a melhor estimativa de erro, é indicado usar dez partições [Witten et al., 2016].

\section{Resultados}

Após a realização das etapas da metodologia, os algoritmos de classificação e a análise dos resultados foram realizados. Assim, esta seção apresenta a avaliação dos algoritmos quanto à sua capacidade de classificar e diferenciar os sentimentos expressos nos tweets. Especificamente, são apresentadas as palavras identificadas como relevantes para identificar cada sentimento (Seção 4.1) e os resultados de uma análise comparativa dos algoritmos de classificação (Seção 4.2). 
Tabela 2. Palavras consideradas relevantes para a análise de cada sentimento. As cores representam palavras semelhantes encontradas em classes diferentes.

\begin{tabular}{|c|l|l|l|l|}
\hline$\#$ & \multicolumn{1}{c|}{ Amor } & \multicolumn{1}{c|}{ Feliz } & \multicolumn{1}{c|}{ Triste } & \multicolumn{1}{c|}{ Chateado } \\
\hline 1 & ECoracao & ESorridente & ERostoFranzido & ERostoDesanimado \\
\hline 2 & ESorridente & ECoracao & EChorando & ERostoFranzido \\
\hline 3 & Amor & Boas & ERostoDesanimado & Tristeza \\
\hline 4 & \#Love & Alegria & Triste & ESemExpressao \\
\hline 5 & Amamos & Agradecer & Acabando & Poxa \\
\hline 6 & \#Paixao & Especial & Coitado & Triste \\
\hline 7 & Amado & Feliz & ESemExpressao & Raiva \\
\hline 8 & Desejo & Sorriso & Infelizmente & Ruim \\
\hline 9 & Flores & Sucesso & Acabaram & Problemas \\
\hline 10 & Fofura & Excelente & Aff & Sozinho \\
\hline
\end{tabular}

\subsection{Relevância das Palavras em Cada Sentimento}

Uma das formas de entender os resultados dos algoritmos é analisar quais elementos foram importantes para a detecção dos sentimentos. A Tabela 2 apresenta as dez principais palavras que foram determinantes para a diferenciação dos sentimentos ao aplicar os algoritmos de classificação. As palavras estão ordenadas pela sua relevância e algumas delas se repetem em determinados sentimentos. Por exemplo, os emojis: ERostoDesanimado e ERostoFranzido são encontrados no sentimento Triste e Chateado, isso ocorre devido à proximidade dos dois sentimentos. Quando o sentimento chateado é expresso, logo, o sentimento de tristeza também pode ser expresso, pois são sentimentos relacionados. Essa proximidade também acontece nos sentimentos Amor e Feliz, com os emojis ECoracao e ESorridente, por exemplo.

Foram considerados quatro mil tweets para cada relação, sendo dois mil para cada sentimento da relação. Essa equiparação foi feita para que os algoritmos classificassem os sentimentos com a mesma quantidade de instâncias, evitando resultados influenciados pelo desbalanceamento das classes, ou seja, uma classe ter uma quantidade de instâncias excessivamente maior que a outra.

\subsection{Análise Comparativa dos Algoritmos de Classificação}

Com base na aplicação dos algoritmos e da divisão das diversas classes de sentimentos, alguns algoritmos se destacaram na classificação, já outros não apresentaram bons resultados. Em geral, os algoritmos baseados no modelo de Bayes foram os que apresentaram os melhores resultados.

Considerando o maior valor de acerto para combinação de classes que representam sentimentos semelhantes (positivo $\mathrm{x}$ positivo e negativo $\mathrm{x}$ negativo) $\mathrm{e}$ distintos (positivo x negativo), as taxas de acerto de classificação de sentimentos foram $85,54 \%$ para o Naive Bayes Multinominal Updateable, $85,41 \%$ para o Naive Bayes Multinominal e 85,64\% para Complement Naive Bayes. 
Tabela 3. Taxa de acerto dos melhores algoritmos para detectar sentimentos positivos $\mathrm{x}$ negativos.

\begin{tabular}{|c|c|c|c|c|}
\hline Algoritmo & $\begin{array}{c}\text { Amor x } \\
\text { Triste }\end{array}$ & $\begin{array}{c}\text { Feliz x } \\
\text { Triste }\end{array}$ & $\begin{array}{c}\text { Feliz x } \\
\text { Chateado }\end{array}$ & $\begin{array}{c}\text { Taxa de Acerto } \\
\text { Média }\end{array}$ \\
\hline $\begin{array}{c}\text { Naive Bayes } \\
\text { Multinominal } \\
\text { Updateable }\end{array}$ & $85,54 \%$ & $81,35 \%$ & $79,60 \%$ & $82,16 \%$ \\
\hline $\begin{array}{c}\text { Naive Bayes } \\
\text { Multinominal }\end{array}$ & $85,41 \%$ & $81,02 \%$ & $79,60 \%$ & $82,01 \%$ \\
\hline $\begin{array}{c}\text { Complement } \\
\text { Naive Bayes }\end{array}$ & $85,64 \%$ & $80,34 \%$ & $79,54 \%$ & $81,84 \%$ \\
\hline
\end{tabular}

As relações compostas por sentimentos distintos positivos $\mathrm{x}$ negativos apresentaram melhores resultados. Os três algoritmos responsáveis por tal desempenho são apresentados na Tabela 3. Em primeiro lugar, a relação Amor x Triste teve 85,64\% de acerto na classificação com o método Complement Naive Bayes (a Tabela 4 apresenta resultados para todos os algoritmos de classificação aplicados para essa relação, o que permite observar as métricas F-Measure e $\mathrm{ROC}^{2}$ com valores maiores também para Complement Naive Bayes em relação a outros algoritmos). Uma das razões para esse resultado é a quantidade de emojis encontrados na relação. Os emojis Ecoracao e Esorridente foram encontrados diversas vezes no sentimento amor. Já no sentimento triste, não foi detectado nenhuma ocorrência desses emojis, conforme mostra a Tabela 2. Em seguida, a relação Feliz x Triste obteve 81,35\% de acerto com o método Naive Bayes Multinominal Updateable. Os emojis ECoração e ESorridente encontrados na classe feliz, também não são localizados na classe triste. Por fim, a relação Feliz x Chateado ficou em terceiro lugar, com $79,60 \%$ de acerto na classificação, com os algoritmos Naive Bayes Multinominal e Naive Bayes Multinominal Updateable. Vale ressaltar que as palavras relevantes para a classificação dos sentimentos positivos $\mathrm{x}$ negativos não se repetem. Consequentemente, essas relações são as que apresentam os melhores resultados comparado com as demais.

Em contrapartida, as relações compostas por sentimentos parecidos positivos $\mathrm{x}$ positivos ou negativos $\mathrm{x}$ negativos apresentam os piores resultados. Os algoritmos usados para a classificação não são capazes de distinguir ou encontrar alguma palavra que expresse um sentimento de maneira que diferencie um do outro. Como consequência, os resultados das relações representadas por sentimentos semelhantes são inferiores às relações compostas por sentimentos distintos. Ademais, nos sentimentos triste e chateado, quatro das dez palavras consideradas relevantes para a classificação se repetem, sendo elas: ERostoFranzido, ESemExpressao, Triste e ERostoDesanimado. Isso indica a semelhança entre os sentimentos, refletindo nos resultados obtidos.

A Tabela 5 mostra a taxa de acerto dos três algoritmos que apresentam os melhores resultados nas relações de sentimentos semelhantes. O método Naive Bayes Multinominal Updateable obteve melhor resultado dos algoritmos aplicados na relação

\footnotetext{
${ }^{2}$ F-Measure and ROC (Receiver Operating Characteristic) são métricas comumente utilizadas para avaliar a qualidade de algoritmos de aprendizagem de máquina.
} 
Tabela 4. Resultado da aplicação dos algoritmos na relação Amor x Triste.

\begin{tabular}{|c|c|c|c|c|c|c|}
\hline \multirow[t]{2}{*}{ Algoritmo } & \multicolumn{2}{|c|}{ Amor } & \multicolumn{2}{|c|}{ Triste } & \multirow[b]{2}{*}{$\begin{array}{l}\text { Acerto } \\
(\%)\end{array}$} & \multirow[b]{2}{*}{$\begin{array}{l}\text { Erro } \\
(\%)\end{array}$} \\
\hline & F-Measure & $\begin{array}{l}\text { ROC } \\
\text { Area }\end{array}$ & F-Measure & $\begin{array}{l}\text { ROC } \\
\text { Area }\end{array}$ & & \\
\hline Naive Bayes & 0,59 & 0,87 & 0,77 & 0,87 & 70,72 & 29,27 \\
\hline $\begin{array}{l}\text { Naive Bayes } \\
\text { Multinominal }\end{array}$ & 0,84 & 0,92 & 0,86 & 0,92 & 85,41 & 4,58 \\
\hline $\begin{array}{l}\text { Naive Bayes } \\
\text { Multinominal } \\
\text { Updateable }\end{array}$ & 0,84 & 0,92 & 0,86 & 0,92 & 85,54 & 4,45 \\
\hline $\begin{array}{l}\text { Sparge Generative } \\
\text { Model }\end{array}$ & 0,82 & 0,92 & 0,84 & 0,92 & 83,84 & 6,15 \\
\hline DMNB Text & 0,82 & 0,92 & $\mathbf{0 , 8 6}$ & 0,92 & 84,50 & 5,49 \\
\hline $\begin{array}{l}\text { Complement Naive } \\
\text { Bayes }\end{array}$ & 0,84 & 0,85 & $\mathbf{0 , 8 6}$ & 0,85 & 85,64 & 4,35 \\
\hline $\begin{array}{l}\text { Bayesian Logistic } \\
\text { Regression }\end{array}$ & 0,79 & 0,80 & 0,80 & 0,80 & 80,38 & 9,61 \\
\hline IBK & 0,76 & 0,83 & 0,69 & 0,83 & 73,59 & 6,40 \\
\hline Ramdom Forest & 0,82 & 0,91 & 0,82 & 0,91 & 83,11 & 6,88 \\
\hline Ramdom Committee & 0,80 & 0,88 & 0,80 & 0,88 & 81,03 & 18,96 \\
\hline
\end{tabular}

Tabela 5.Taxa de acerto dos algoritmos para sentimentos negativos $\mathrm{x}$ negativos $\mathrm{e}$ positivos $\mathrm{x}$ positivos.

\begin{tabular}{|c|c|r|r|}
\hline Algoritmo & $\begin{array}{c}\text { Amor x } \\
\text { Feliz }\end{array}$ & $\begin{array}{c}\text { Triste } \mathbf{x} \\
\text { Chateado }\end{array}$ & $\begin{array}{c}\text { Taxa de Acerto } \\
\text { Média }\end{array}$ \\
\hline $\begin{array}{c}\text { Naive Bayes Multinominal } \\
\text { Updateable }\end{array}$ & $73,59 \%$ & $66,48 \%$ & $70,03 \%$ \\
\hline Naive Bayes Multinominal & $73,25 \%$ & $65,16 \%$ & $69,20 \%$ \\
\hline Complement Naive Bayes & $72,95 \%$ & $64,14 \%$ & $68,54 \%$ \\
\hline
\end{tabular}


relação Chateado x Triste, com $66,48 \%$ de classificação correta. Já nos sentimentos feliz e amor, os emojis: ECoracao e ESorridente aparecem em ambos sentimentos, indicando a proximidade entre eles. Apesar da semelhança, a relação Amor x Feliz obteve um resultado de $73,59 \%$ de classificação correta com o método Naive Bayes Multinominal Updateable, sendo superior ao resultado obtido pela relação Triste x Chateado.

Os algoritmos IBK, Random Forest, Random Committee não apresentam resultados eficientes quando comparados com os resultados apresentados no trabalho feito por Garg e Khurana [2014]. Isso se dá pelo fato do formato dos dados serem diferentes e em outro idioma. Outro ponto importante que distingue os dois trabalhos é a quantidade de dados usados para as etapas de processamento e teste. Em Garg e Khurana (2014) foram considerados cerca de 142.000 para o conjunto de treino e teste.

Além das etapas de treino e de processamento dos dados, que foram importantes para a classificação dos sentimentos, os emojis se mostram essenciais para a classificação. Tais ideogramas representam as principais palavras para caracterizar um sentimento, diferenciando um sentimento positivo de outro negativo.

\section{Conclusão}

Neste trabalho, foram utilizados métodos de classificação baseados no modelo Naive Bayes e em outros modelos de classificação, como árvores de decisão, para classificar tweets em português brasileiro. Apesar de ser um tema popular, ainda existem vários desafios para a descoberta de sentimentos, especialmente, em textos na língua portuguesa. O estudo considerou sete sentimentos: triste, chateado, amor, feliz, raiva, inveja, ironia. Os dados foram obtidos do Twitter, no qual foram coletados 12.814 tweets. Ao final do processamento, inveja, ironia e raiva foram excluídos, restando

9.631 tweets. Após a coleta, os dados foram separados em relações de sentimentos: positivo $\mathrm{x}$ negativo, negativo $\mathrm{x}$ negativo e positivo $\mathrm{x}$ positivo, para, assim, serem aplicados os algoritmos de classificação. Os resultados mostraram que os algoritmos baseados no modelo Naive Bayes apresentaram melhor acurácia. As relações que possuem os sentimentos positivos $\mathrm{x}$ negativos foram as que obtiveram os melhores resultados, chegando a $85 \%$ de acerto na classificação com o Complement Naive Bayes. Apesar dos métodos produzirem resultados inferiores quando aplicados no português brasileiro, os resultados foram satisfatórios e de acordo com outras pesquisas na área.

Para os trabalhos futuros, planeja-se ampliar a coleta de dados, aplicar mais métodos de classificação e comparar com resultados obtidos em textos na língua inglesa. Ademais, métodos de análise de sentimentos podem ser utilizados a fim de realizar uma análise mais detalhada. Por exemplo, explorar a importância dos emojis e emoticons na identificação dos sentimentos expressos e detectar múltiplos sentimentos.

Agradecimentos. Este trabalho foi parcialmente financiado pelo CNPq.

\section{Referências}

Araújo, M.; Gonçalves, P.; Benevenuto, F. (2015). Métodos para Análise de Sentimentos em Mídias Sociais. In Procs. of Simpósio Brasileiro de Multimídia e Web (WEBMEDIA), p. 27 - 30, Manaus, Brasil. 
Araújo, M.; Reis, J.; Pereira, A.; Benevenuto, F. (2016). An Evaluation of Machine Translation for Multilingual Sentence-level Sentiment Analysis. In Procs. of ACM Symposium on Applied Computing (SAC), p. 1140 - 1145, Pisa, Itália.

Bouazizi, M.; Ohtsuki, T. (2016). Sentiment Analysis in Twitter: From Classification to Quantification of Sentiments within Tweets. In Procs. of Global Communications Conference (GLOBECOM). p. 1 - 6, Washington, USA.

Caetano, J. A. C.; Lima, H. S. L.; dos Santos Santos, M. F.; Marques-Neto, H. T. M. N. (2017). Utilizando Análise de Sentimentos para Definição da Homofilia Política dos Usuários do Twitter durante a Eleição Presidencial Americana de 2016. In Procs. of Brazilian Workshop on Social Network Analysis and Mining (BraSNAM), p. 480-491, São Paulo, Brasil.

Cavalcanti, D. C. et al. (2012). Análise de Sentimento em Citações Científicas para Definição de Fatores de Impacto Positivo. In Procs. of International Workshop on Web and Text Intelligence (WTI), 4. p. 1 - 10, Paraná, Brasil.

Dwivedi, S.; Kasliwal, P.; Soni, S. (2016). Comprehensive study of data analytics tools (RapidMiner, Weka, R tool, Knime). In Procs. of Symposium On Colossal Data Analysis And Networking (CDAN), p. 1 - 8, Indore, India.

França, Tiago C. de; Oliveira, Jonice. (2014). Análise de Sentimento de Tweets Relacionados aos Protestos que ocorreram no Brasil entre Junho e Agosto de 2013. In Procs. of Brazilian Workshop on Social Network Analysis and Mining (BraSNAM), p. 128 - 139, Brasília, Brasil.

Garg, T.; Khurana, S. S. (2014). Comparison of classification techniques for intrusion detection dataset using WEKA. In Procs. of International Conference on Recent Advances and Innovations in Engineering (ICRAIE). p. 1 - 5, Jaipur, India.

Garimella, Kiran. et al. (2018). Quantifying Controversy in Social Media. Journal ACM Transactions on Social Computing, v. 1, n. 1, p. 3:1--3:27.

Matsuda, Y. (2017). Development of Emotion Teaching Interfaces using Emoticons and Emojis. In Procs of International Conference On Intelligent Human-Machine Systems and Cybernetics (IHMSC), 9, p. 253 - 258, Hangzhou, China.

Neri, F; Aliprandi, C; Capeci, F; Cuadros, M. (2012) Sentiment Analysis on Social Media. In Procs. of IEEE/ACM International Conference on Advances in Social Networks Analysis and Mining (ASONAM), p. 919 - 926, Istanbul, Turquia.

Pandey, A. K.; Rajpoot, D. S. (2016). A comparative study of classification techniques by utilizing WEKA. In Procs. of International Conference On Signal Processing And Communication (ICSC). p. 219 - 224, Bangalore, India.

Witten I. H.; Frank, E; Hall, M. A.; Pal, C. J. (2016). Data Mining: Practical Machine Learning Tools and Techniques. Morgan Kaufmann Publishers, $4^{\text {th }}$ edition. ISBN: 978-0128042915.

Yadollahi, A.; Shahraki, A. G.; Zaiane, O. R. (2017). Current State of Text Sentiment Analysis from Opinion to Emotion Mining. ACM Computing Surveys, v. 50, n.2, p. 1 -33 . 